\title{
Conocimiento sobre VIH-SIDA en estudiantes de secundaria de Cartagena, Colombia
}

\author{
Secondary students' knowledge about HIV-AIDS (Cartagena, Colombia) \\ Edna Gómez-Bustamante y Zuleima Cogollo-Milanés
}

Grupo cuidado a la salud de los colectivos. Facultad de Enfermería, Universidad de Cartagena. Colombia.egomezb@unicartagena.edu.co,zcogollom@unicartagena.edu.co

Recibido 5 Enero 2011/Enviado a Modificación 15 Julio 2011/Aceptado 21 Septiembre 2011

\section{RESUMEN}

Objetivo Determinar el nivel de conocimiento en VIH-SIDA en estudiantes de Cartagena, Colombia.

Método Se diseñó un estudio transversal con una muestra de estudiantes de secundaria que completó el cuestionario de seis preguntas sobre conocimientos y mitos relacionados con VIH-Sida. Mediante regresión logística se ajustaron asociaciones.

Resultados Participaron 2625 estudiantes entre 10 y 20 años. Un total $249(9,5 \%$ IC95 \% 8,4-10,6) respondieron en forma correcta el cuestionario. Ser estudiante de media vocacional (OR 2,62; IC95 \% 4,82-13,29) y estudiar en colegio privado (OR 1,32; IC95 \% 1,24-3,54), ajustados por edad, se asociaron a buen conocimiento en VIH-Sida.

Conclusiones Los estudiantes de secundaria de Cartagena presentan un bajo conocimiento sobre el VIH-Sida. El nivel es superior en estudiantes de media vocacional y de colegios privados. Se necesita más investigación y fortalecer la formación en educación en salud sexual y reproductiva.

Palabras Clave: Síndrome de inmunodeficiencia adquirida, actitud, adolescente, estudiantes, estudios transversales (fuente: DeCS, BIREME).

\section{ABSTRACT}

Objective Determining the level of HIVIAIDS knowledge amongst high-students living in Cartagena, Colombia.

Method A cross-sectional study was designed using a sample of secondary school students who completed a six-question questionnaire about their knowledge of HIVIAIDS and the myths related to it. Logistic regression was used for adjusting associations.

Results A total of 2,625 10- to 20-year-old students participated in the research. A group of $249(9.5 \mathrm{OR} ; 8.4-10.695 \% \mathrm{Cl})$ answered the questionnaire properly. 
Being a high-school student (2.62 OR; 4.82-13.29 $95 \% \mathrm{Cl}$ ) and studying in a private school (1.32 OR, 1.24 to $3.5495 \% \mathrm{Cl}$ ), adjusted for age, were associated with having good HIVIAIDS knowledge.

Conclusions Students from secondary schools in Cartagena were seen to have limited knowledge of HIVIAIDS. A higher level of knowledge was observed amongst high school and private school students. More research is needed to strengthen sexual and reproductive health education.

Key Words: Acquired immunodeficiency syndrome, attitude, adolescent, student, cross-sectional study (source: $\mathrm{MeSH}, \mathrm{NLM}$ ).

$\mathrm{E}$ n todo el mundo, la infección por el virus de la inmunodeficiencia humana (VIH) y el Síndrome de Inmunodeficiencia (Sida) representan un problema prioritario de salud pública que afecta un número importante de adolescentes (1).

La educación en salud es una estrategia importante en la prevención de la transmisión del VIH-Sida (2). Sin embargo, los adolescentes muestran un conocimiento limitado en el área (3-10). La Organización Mundial de la Salud diseñó un instrumento para unificar la evaluación del conocimiento en VIHSida en adolescentes (11). Con una versión adaptada del cuestionario, Pérez et al, hallaron que sólo un tercio de un grupo de estudiantes chilenos respondió correctamente todo el cuestionario; el conocimiento fue superior en estudiantes de colegios privados e independiente de la edad, del sexo y del nivel de escolaridad (12).

En Colombia, no hay estudios que informen el nivel de conocimiento en VIH-Sida en estudiantes de secundaria. Es importante explorar los conocimientos básicos en VIH-Sida de los estudiantes colombianos con el ánimo de fortalecer la educación en el área y reducir con ello el riesgo de infección (2).

El objetivo de este estudio fue determinar el nivel de conocimiento en VIHsida en estudiantes de Cartagena, Colombia.

\section{MÉTODOS}

Se diseñó un estudio transversal que aprobó el Comité de Ética en Investigación de la Universidad de Cartagena. Los representantes legales de los estudiantes menores de edad firmaron consentimiento informado. Los estudiantes asintieron participar. Se siguieron las normas colombianas para la investigación en salud (13). 
Se estimó una muestra inicial de 1300 estudiantes para una prevalencia esperada de conocimiento adecuado en VIH-sida de 32,5\% con base en la prevalencia que se encontró en un estudio chileno (12). El margen de error fue $2,5 \%$ y un nivel de confianza de $95 \%$. Se hizo una reposición de 65 participantes (5\%) por no respuesta. Para explorar asociaciones con aceptable intervalo de confianza se duplicó la muestra a 2730 participantes.

Los estudiantes diligenciaron en el aula de clases un instrumento modificado de seis preguntas para conocimiento y mitos relacionados con el VIH-sida a partir del cuestionario de cinco puntos que diseñó la Organización Mundial de la Salud. Al cuestionario se le pueden hacer ajustes, según las características de las personas participantes (11). Se usó la versión de seis puntos que aplicaron Pérez et al., en un estudio antes citado para contar con una medición que permitiera una comparación más confiable (12). Las tres primeras exploraron el ABC sobre la prevención en VIH (Abstinencia, abstinence; fidelidad, be fairful; y uso de condón, condom use) y; las tres últimas cuantificaron conocimientos erróneos. Se consideró conocimiento adecuado en prevención las respuestas correctas a las respectivas tres preguntas, conocimiento adecuado en mitos a respuestas correctas a las tres preguntas respectivas y conocimiento adecuado global a la respuesta correcta a las seis preguntas (11).

Se realizó un análisis descriptivo de la información, se estimaron media y desviación estándar (DE) para variables cuantitativas y frecuencias y porcentajes para variables cualitativas nominales. Para el análisis bivariado se dicotomizaron todas las variables; se estimaron razones de oportunidad (OR) con intervalos de confianza del $95 \%$ (IC95\%). Para el análisis multivariado se siguieron las recomendaciones de Greenland (14). Al modelo final se le estimó la bondad del ajuste de Hosmer-Lemeshow, se esperó un valor de $\mathrm{p}$ mayor de 0,10 (15). Todos los cálculos se hicieron en STATA 9.0 (16).

\section{RESULTADOS}

Participaron 2625 estudiantes entre 10 y 20 años, con una media de 13,8 años $(\mathrm{DE}=2,0)$, residentes en estrato socioeconómico de I a VI. En la tabla 1 se presentan las características demográficas de la población. En la tabla 2 se muestran las frecuencias y porcentajes de respuestas correctas en el cuestionario sobre conocimiento en VIH-Sida. Frecuencias y porcentajes en conocimiento en estudiantes de secundaria en Cartagena, Colombia. Un total de 1008 estudiantes $(38,4 \%$; IC95\% 36,5-40,3) respondieron correctamente las preguntas en prevención; 530 (20,2\% IC95 \% 18,7-21,7) dieron una 
correcta respuesta a las preguntas relacionadas con los mitos; y 249 (9,5\% IC95 \% 8,4-10,6) respondieron en forma correcta todo el cuestionario.

Tabla 1. Frecuencias y porcentajes de las variables estudiadas en estudiantes de secundaria en Cartagena, Colombia

\begin{tabular}{lcc}
\hline Variables & $\mathrm{n}$ & $\%$ \\
\hline Edad de 10 a 15 años & 1585 & 60,4 \\
Edad de 16 a 20 años & 1040 & 39,6 \\
Sexo femenino & 1425 & 54,3 \\
Sexo masculino & 1200 & 45,7 \\
Básica secundaria & 1778 & 67,7 \\
Media vocacional & 847 & 32,3 \\
Nivel socioeconómico bajo & 1911 & 72,8 \\
Nivel socioeconómico medio-alto & 714 & 27,2 \\
Colegio público & 1817 & 69,2 \\
Colegio privado & 808 & 30,8 \\
Heterosexuales & 2411 & 91,9 \\
No heterosexuales (gays, lesbianas e inseguros) & 214 & 8,1 \\
Alguna relación sexual alguna vez en la vida & 587 & 22,4 \\
Sin experiencia sexual & 2038 & 77,6 \\
Información sexual en el colegio & 1586 & 60,4 \\
No información sexual en el colegio & 1039 & 39,6 \\
\hline
\end{tabular}

Tabla 2. Frecuencias y porcentajes de respuestas correctas en el cuestionario sobre conocimiento en VIH-Sida en estudiantes de secundaria en Cartagena, Colombia

\begin{tabular}{|c|c|c|c|}
\hline Pregunta & $\mathrm{N}$ & $\%$ & IC95\% \\
\hline $\begin{array}{l}\text { ¿Puede reducirse el riesgo de transmisión del } \mathrm{VIH} \text { si } \\
\text { la persona se abstiene de tener relaciones } \\
\text { sexuales? }\end{array}$ & 1702 & 64,8 & $63,0-66,6$ \\
\hline $\begin{array}{l}\text { ¿Es posible reducir el riesgo de transmisión del VIH } \\
\text { si tiene relaciones sexuales sólo con un compañero } \\
\text { fiel que no esté contagiado? }\end{array}$ & 1553 & 59,2 & $57,3-61,1$ \\
\hline $\begin{array}{l}\text { ¿Es posible reducir el riesgo de transmisión del VIH } \\
\text { con el uso de condones? }\end{array}$ & 1655 & 63,1 & $62,3-65,0$ \\
\hline $\begin{array}{l}\text { ¿Es posible que una persona que parece sana esté } \\
\text { infectada con el VIH? }\end{array}$ & 1865 & 71,1 & $69,4-72,8$ \\
\hline $\begin{array}{l}\text { ¿Es posible que una persona se contagie del } \mathrm{VIH} \text { a } \\
\text { través de picaduras de mosquitos? }\end{array}$ & 1540 & 58,7 & $56,8-60,6$ \\
\hline $\begin{array}{l}\text { ¿Es posible que una persona se contagie del VIH si } \\
\text { se comparte baños públicos con alguien que está } \\
\text { contagiado? }\end{array}$ & 1289 & 49,1 & $47,2-51,0$ \\
\hline
\end{tabular}

En el análisis bivariado se observó que edad entre 16 y 20 años, ser estudiante de media vocacional, residir en estrato socioeconómico medio o alto, estudiar en colegio privado, reconocerse heterosexual y recibir información sobre VIH en el colegio se relacionaron con mejor conocimiento global en VIH-sida. Las razones de disparidad se presentan en la Tabla 3. Sin embargo, en el modelo multivariado sólo ser estudiante de media vocacional y estudiar en colegio privado, ajustado por edad, se mantuvieron en el rango significativo. El modelo se presenta en la Tabla 4. 
Tabla 3. Asociaciones entre variables estudiadas y el conocimiento

\begin{tabular}{lcc}
\multicolumn{3}{l}{ adecuado global en VIH-sida en estudiantes de Cartagena, Colombia } \\
\hline Variables & OR & IC95\% \\
\hline Edad de 16 a 20 años & 1,55 & $1,20-2,02$ \\
Edad de 10 a 15 años & 1,00 & - \\
Sexo masculino & 0,90 & $0,69-1,17$ \\
Sexo femenino & 1,00 & - \\
Estudiante de media vocacional & 2,18 & $1,68-2,85$ \\
Estudiante de básica secundaria & 1,00 & - \\
Nivel socioeconómico medio-alto & 1,35 & $1,02-1,79$ \\
Nivel socioeconómico bajo & 1,00 & - \\
Colegio privado & 1,33 & $1,01-1,75$ \\
Colegio público & 1,00 & - \\
Heterosexuales & 2,02 & $1,09-3,76$ \\
No heterosexuales (gays, lesbianas e inseguros) & 1,00 & - \\
Alguna relación sexual alguna vez en la vida & 1,31 & $0,98-1,77$ \\
Sin experiencia sexual & 1,00 & - \\
Información sexual en el colegio & 1,46 & $1,10-1,93$ \\
No información sexual en el colegio & 1,00 & - \\
\hline
\end{tabular}

Tabla 4. Modelo de regresión logística para el conocimiento adecuado global en VIH-sida en estudiantes de Cartagena, Colombia

\begin{tabular}{lcc}
\hline Variable & OR & IC95\% \\
\hline Estudiante de media vocacional & 2,62 & $4,82-13,29$ \\
Colegio privado & 1,32 & $1,24-3,54$ \\
Edad de 16 a 20 años & 0,79 & $0,53-1,17$ \\
\hline Prueba de Hosmer-Lemeshow con chi cuadrado = 1,02; grados de libertad = 5; probabilidad =
\end{tabular}

0,795

\section{DISCUSIÓN}

En el presente estudio se observa que aproximadamente el $10 \%$ de los estudiantes de secundaria de Cartagena presenta un adecuado conocimiento en VIH-Sida. Los estudiantes de media vocacional y de colegios privados informaron mayor conocimiento.

El nivel de conocimiento en VIH-sida varía ampliamente en adolescentes estudiantes, según el contexto sociocultural y el instrumento de medición (310). En la presente investigación se observó que el 9,5\% de los estudiantes informaron un adecuado conocimiento en este tópico. El único estudio que informó el nivel de conocimiento en VIH-sida con el mismo instrumento de medición lo realizaron Pérez et al., quienes observaron que el 32,5\% de los estudiantes respondió en forma correcta el cuestionario (12).

De la misma manera, en el presente estudio se encontró que los estudiantes de colegios privados y mayor escolaridad respondieron mejor y el conocimiento fue independiente de la edad, el sexo, nivel socioeconómico, orientación sexual, 
informar relaciones sexuales y recibir información sobre VIH-Sida en el contexto escolar. Pérez et al., hallaron sólo mayor conocimiento en estudiantes de colegios privados, sin considerar alguna relación con la edad, el sexo y el nivel de escolaridad (12).

De lo anterior, es importante destacar que un número de adolescentes inicia relaciones coitales sin conocer claramente las formas de transmisión y de prevención adecuada para VIH-Sida. Campo-Arias y colaboradores documentaron que informar relaciones sexuales se asoció a no información sobre $\mathrm{VIH}$, como parte del currículo académico, sólo en el grupo de adolescentes mujeres de Santa Marta, Colombia (17).

En adolescentes estudiantes, es necesario fortalecer la educación en salud sexual y reproductiva con una visión integral que permita la adopción de conocimientos y valores personales y prosociales que se expresen a diario en comportamientos saludables, de autocuidado, de respeto de la propia integridad física y de la pareja (18). De igual manara, es importante mejorar la formación de los docentes en esta área dado que los estudiantes reciben la mayor información sobre VIH-Sida en el contexto escolar $(9,19)$.

Este es el primer estudio que informa el nivel de conocimiento sobre VIHsida en una muestra representativa de estudiantes de secundaria de Cartagena, Colombia. No obstante, presenta las limitaciones propias de los estudios transversales que no permiten definir la relación causa-efecto (20).

Los estudiantes de secundaria de Cartagena presentan un limitado conocimiento sobre VIH-Sida. El nivel es superior en estudiantes de media vocacional y de colegios privados. Se necesita más investigación y fortalecer la formación en educación en salud sexual y reproductiva

Agradecimiento: La Vicerrectoría de Investigación de la Universidad de Cartagena financió este estudio, según acta de compromiso (054-2009). Agradecemos a Adalberto Campo-Arias, MD, del Instituto de Investigación del Comportamiento Humano, por la asesoría para la realización de este informe.

\section{REFERENCIAS}

1. Singh HK, Gupta A, Siberry GK, Gupte K, Sastry J, Kinikar A. The Indian pediatric HIV epidemic. Curr HIV Res. 2008; 6: 419-432.

2. Malow RM, Kershaw T, Sipsman H, Rosenberg R, Dévieux JG. HIV prevention interventions for adolescents: A look back and ahead. Curr HIVIAIDS Report. 2007; 4: 173-180. 
3. Amirkhanian YA, Tiunov DV, Kelly JA. Risk factors for HIV and other sexually transmitted diseases among adolescents in St. Petersburg, Russia. Fam Plan Persp. 2001; 33: 106-112.

4. Merakou K, Costopoulos C, Marcopoulos J, Kourea-Kremastinou J. Knowledge, attitudes and behavior after 15 years of HIVIAIDS prevention in schools. Eur J Public Health. 2002; 12: 90-93.

5. Villaseñor-SierraA, Caballero-Hoyos R, Hidalgo-San Martín A, Santos-Preciado JI. Conocimiento objetivo y subjetivo sobre el VIH/SIDA como predictor de uso de condón en adolescentes. Salud Publica Mex. 2003; 45 (supl. 1): S73-S80.

6. Savaser S. Knowledge and attitudes of high school students about AIDS: A Turkish perspective. Public Health Nurs. 2003; 20: 71-79.

7. Tavcosi A, Zaferani A, Enzevaei A, Tajik P, Ahmadinezhad Z. Knowledge and attitude towards HIVIAIDS among Iranian students. BMC Public Health. 2004; 4: 17.

8. Turhan E, Inandi $Y$, Inandi T. Risk perception, knowledge and social distance of Turkish high school students about HIVIAIDS. J Public Health. 2006; 28: 137-138.

9. Catacora-López F, Villanueva-Roque J. Conocimientos sobre VIH/SIDA y prácticas sexuales de escolares de Tacna, Perú 2004. Rev Peru Med Exp Salud Publica. 2007; 24: 240247.

10. Dávila ME, Tagliaferro AZ, Bullones X, Daza D. Niveles de conocimiento de adolescentes sobre VIH/SIDA. Rev. Salud Publica (Bogotá) 2008; 10: 716-722.

11. Una guía de indicadores para monitorear y evaluar los programas en prevención del VIH/ SIDA. Ginebra: Organización Mundial de la Salud; 2005.

12. Pérez R, Barrales I, Jara J, Palma V, Ceballos A. Knowledge of HIVIAIDS among adolescents in Chillán, Chile. Midwifery. 2008; 24: 503-508.

13. Resolución 008430 por la cual se establecen las normas científicas, técnicas y administrativas para la investigación en salud. Bogotá: Ministerio de Salud de Colombia; 1993.

14. Greenland S. Modeling and variable selection in epidemiologic analysis. Am J Public Health 1989; 79: 340-9.

15. Hosmer DW, Lemeshow S. Applied logistic regression. 2nd edition. New York: John Wiley \& Sons Inc; 2000

16. STATA 9.0 for windows. College Station: StataCorp LP; 2005.

17. Campo-Arias A, Ceballos-Ospino GA, Herazo $E$. Factores asociados por género a relaciones sexuales en adolescentes de Santa Marta, Colombia, en el año 2004. Rev Colomb Obstet Ginecol. 2008; 59: 276-284.

18. Gallegos EC, Villarruel AM, Loveland-Cherry C, Runis DL, Zhou Y. Intervención para reducir riesgo en conductas sexuales de adolescentes: un ensayo aleatorizado y controlado. Salud Publica Mex. 2008; 50: 59-66.

19. Caballero R, Villaseñor A, Hidalgo A. Fuentes de información y su relación con el grado de conocimiento sobre el SIDA en adolescentes de México. Rev Saude Publica. 1997; 31: 351-359.

20. Grimes DA, Schulz KF. Bias and causal association in observational research. Lancet. 2002; 359: 248-252. 